\title{
SECAGEM DA MISTURA COLÁGENO HIDROLISADO-POLPA DE UVA EM LEITO DE JORRO: ANÁLISE DA EFICIÊNCIA DE OBTENÇÃO DE PÓ E DA INCORPORAÇÃO DE ANTOCIANINAS NO PRODUTO FINAL
}

\author{
J. J. BUTZGE ${ }^{1}$, F. H. HANAO ${ }^{1}$; F. C. GODOY ${ }^{2}$ e S. C. S. ROCHA ${ }^{1}$ \\ ${ }^{1}$ Universidade Estadual de Campinas, Faculdade de Engenharia Química \\ 2 The University of Queensland, School of Agriculture and Food Sciences \\ E-mail para contato: jjunior@feq.unicamp.br
}

\begin{abstract}
RESUMO - Este trabalho teve o objetivo de desenvolver um novo produto alimentício em pó, de elevado valor nutricional, a partir da secagem de uma composição constituída por colágeno hidrolisado $(\mathrm{CH})$ e polpa de uva. Foi avaliada a influência das condições operacionais de secagem em leito de jorro no desempenho do processo, bem como na composição de antocianinas do produto final. Um planejamento experimental composto central rotacional foi proposto, com as variáveis independentes: temperatura do ar de jorro, concentração de polpa na pasta de secagem e vazão de atomização no leito. As respostas foram: eficiência de produção e teor de antocianinas do pó. Foram obtidas altas eficiências de produção de pó, de até $86,8 \%$. Também foi possível incorporar quantidades significativas de antocianinas no produto final, de até $38,1 \mathrm{mg}$ de antocianinas por $100 \mathrm{~g}$ de pó. A boa relação entre eficiência de produção de pó e preservação dos compostos antociânicos provenientes da fruta no produto final demonstram que o leito de jorro é uma técnica viável para secagem deste tipo de produto. Evidencia-se que a composição alimentícia desenvolvida apresenta elevado potencial de consumo na forma de pó como um produto pronto, ou, alternativamente, como ingrediente para um alimento ou bebida.
\end{abstract}

\section{INTRODUÇÃO}

A preocupação com a saúde, alimentação e qualidade de vida têm alavancado a busca por bebidas e alimentos nutricionalmente enriquecidos, que suplementem a alimentação diária, previnam doenças, auxiliem na beleza ou promovam saúde e bem estar.

O colágeno hidrolisado $(\mathrm{CH})$ é um suplemento alimentar comercializado na forma de cápsulas ou pós, com intuito de estimular a síntese das fibras de colágeno corpóreas. O $\mathrm{CH}$ é atrativo para o consumo devido a fácil absorção e distribuição para a corrente sanguínea e demais tecidos do corpo (Moskowitz, 2000; Walrand et al. 2008); ademais, possui elevado nível de segurança e inocuidade à saúde humana, atestada pelo status de GRAS (generally recognized as safe) (Moskowitz, 2000).

Embora amplamente comercializado, o $\mathrm{CH}$ raramente vem adicionado de aditivos naturais; sobretudo, na forma de um produto alimentício em pó que possua em sua composição $\mathrm{CH}$ agregado a 
polpas de frutas e que preserve os compostos bioativos provenientes da fruta, como as antocianinas.

Relacionados ao combate dos radicais livres, compostos fenólicos como as antocianinas têm ganhado cada vez mais atenção da comunidade científica mundial, devido à reconhecida capacidade antioxidante. A ingestão destes componentes tem sido associada com a redução do risco de doenças cardiovasculares e cancerígenas (Neuhouser, 2004).

Fruto da videira (Vitis vinifera L.), a uva é rica em compostos fenólicos como as antocianinas, flavonóis, taninos e resveratrol (Fanzone et al. 2011). Entende-se, portanto, que existe um amplo potencial de exploração na combinação de polpa de uva com $\mathrm{CH}$ para fabricação de um novo produto alimentício em pó, com elevado valor nutricional agregado e que incorpore os compostos naturais benéficos à saúde bem como características físicas, nutricionais e organolépticas da fruta.

Dentro deste contexto, este trabalho refere-se ao estudo do processo de secagem da mistura $\mathrm{CH}$ e polpa de uva em um secador de leito de jorro, visando o desenvolvimento de um novo produto alimentício em pó de alto valor nutricional. Foram testadas diferentes proporções $\mathrm{CH}$ - polpa de uva na formulação, bem como variadas condições operacionais durante o processo de secagem em leito de jorro. O produto final (pó) foi quantificado e caracterizado, visando encontrar as condições processo que garantam boa eficiência de produção de pó e também minimizem a degradação dos compostos antociânicos, permitindo incorpora-los em quantidades significativas no pó formado.

\section{MATERIAIS E MÉTODOS}

\subsection{Materiais}

A preparação das pastas de secagem consistiu em três etapas: primeiramente dissolveu-se $\mathrm{CH}$ bovino em pó (Gelita, Brasil) em água a $50^{\circ} \mathrm{C}(50 \% \mathrm{~m} / \mathrm{m})$; na segunda etapa a solução foi submetida a cisalhamento em moinho coloidal por $30 \mathrm{~s}$ para evitar a formação de aglomerados; na ultima etapa, a solução de colágeno hidrolisado $(\mathrm{SCH})$ foi misturada com polpa de uva industrializada congelada (De Marchi, Brasil), sob agitação, em cinco proporções (m/m), apresentadas na Tabela 1.

Tabela 1 - Composição das pastas de secagem

\begin{tabular}{|c|c|c|}
\hline Composição & Polpa de uva $(\%)$ & Solução de colágeno hidrolisado (SCH) (\%) \\
\hline A & 8 & 92 \\
\hline B & 25 & 75 \\
\hline C & 50 & 50 \\
\hline D & 75 & 25 \\
\hline E & 92 & 8 \\
\hline
\end{tabular}

\subsection{Caracterizações físico-químicas}

As pastas de secagem foram caracterizadas pelas seguintes análises: pH, método 017/IV do Instituto Adolfo Lutz (2008) através de pHmetro calibrado (DM22, Digimed, Brasil); reologia, 


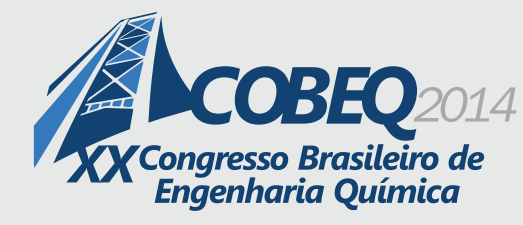

através da curva pseudo-reológica com dados obtidos em um reômetro capilar; conteúdo de umidade, através do método 014/IV, segundo Instituto Adolfo Lutz (2008); conteúdo de antocianinas, calculado pelo método oficial AOAC 2005.02. Para extração das antocianinas foi utilizada uma solução de etanol acidificada com HCl (85:15 v/v), pH=1, segundo método proposto por Fuleki e Francis (1968). O pó resultante do processo foi caracterizado quanto ao teor de umidade e conteúdo de antocianinas.

\subsection{Sistema experimental}

O sistema experimental utilizado nos ensaios de secagem consiste em um leito de jorro conecilíndrico construído em acrílico Plexiglas ${ }^{\circledR}$ para facilitar a visualização da dinâmica das partículas durante o processo. A Figura 1 ilustra o equipamento utilizado e os demais periféricos.

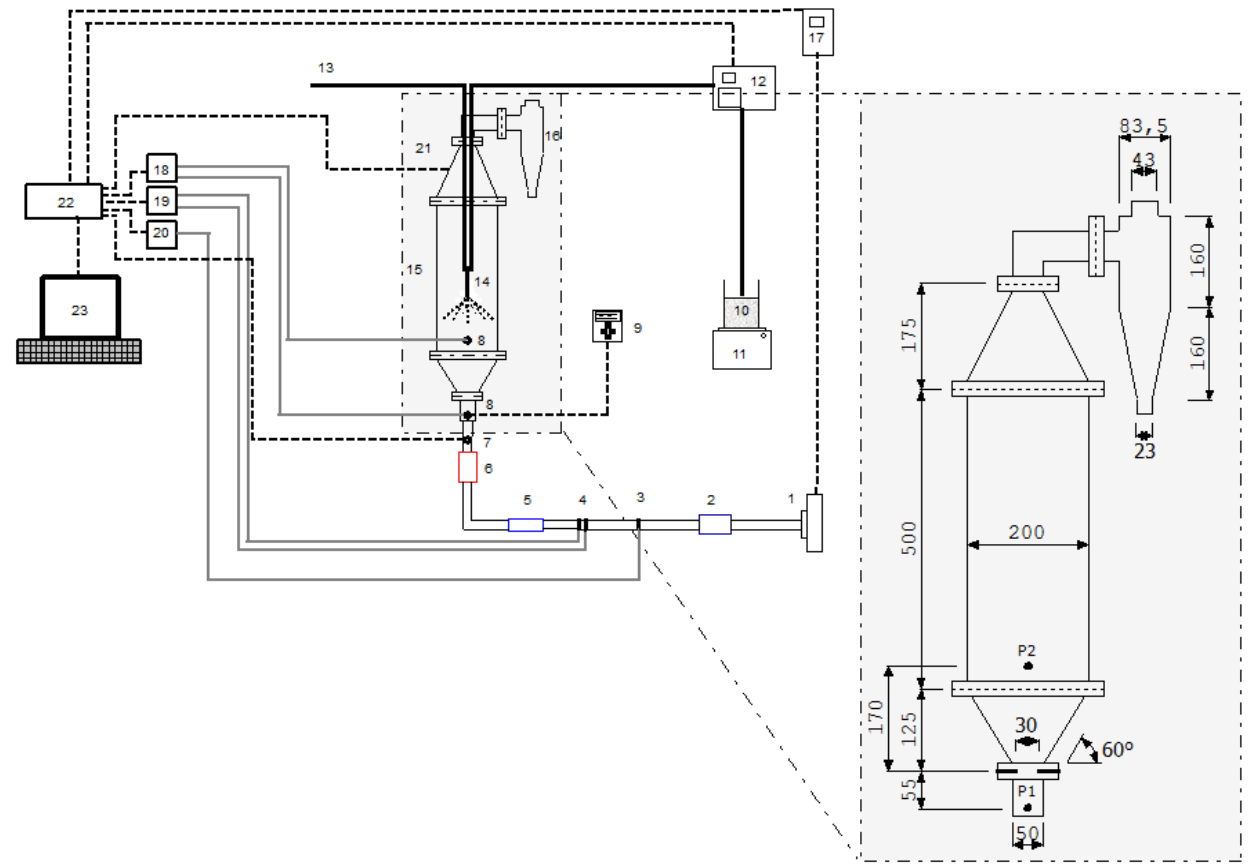
(1) Soprador
(2) resfriador de ar
(3) Pressão estática na linha
(4) Placa de orifício
(5) Leito de sílica gel
(6) Aquecedores de ar
(7) Sensor de temper atura e umidade
(8) Tomadas de pressão no leito
(9) Controlador de temperatura
(10) Pasta de alimentação
(11) Agitador magnético
(12) Bomba peristáltica
(13) Linha de ar comprimido
(14) Bico atomizador duplo fluido
(15) Câmara plenum
(16) Ciclone Lapple
(17) Inversor de frequência
(18) Transdutores de pressão diferencial
(19) Transdutores de pressão diferencial
(20) Transdutor de pressão absoluto
(21) Sensor de temperatura e umidade
(22) Sistema de aquisição de dados
(23) Micropr ocessador.

Figura 1 - Sistema experimental. Dimensões em mm.

Testes preliminares de secagem foram realizados para definir o método de alimentação da pasta (atomização), pressão de atomização (10 psig) e tipo de inerte (polipropileno), enquanto testes fluidodinâmicos definiram a carga de inerte $(1 \mathrm{~kg})$, velocidade de jorro mínimo $\left(\mathrm{U}_{\mathrm{jm}}=0,4 \mathrm{~m} / \mathrm{s}\right)$ bem como a velocidade operacional do ar de jorro $\left(\mathrm{U}_{\mathrm{op}}=0,44 \mathrm{~m} / \mathrm{s}\right)$. Em cada ensaio de secagem foram atomizados $300 \mathrm{~g}$ de pasta. $\mathrm{O}$ sistema era desligado 20 minutos após o termino da atomização.

\subsection{Ensaios de secagem}

Um planejamento experimental composto central com triplicata no ponto central foi delineado para os experimentos de secagem. Temperatura (T), vazão de atomização (W) e percentual de solução de colágeno hidrolisado ( $\mathrm{SCH}$ ) na composição da pasta de secagem foram as variáveis utilizadas. As respostas dos ensaios de secagem foram teor de antocianina (AA) e eficiência de obtenção de pó (E). 
A eficiência de produção de pó ( $\varepsilon$ ) é dada pela Equação 1.

$$
\varepsilon=\frac{M_{\text {pó }}}{Q_{\text {pasta }} C_{s} t}
$$

Onde: $M_{p o ́}=$ massa seca de pó coletada; $Q_{\text {pasta }}=$ vazão volumétrica da pasta atomizada; $C s=$ concentração de sólidos secos; $t=$ tempo de alimentação.

Adicionalmente quantificou-se a retenção de sólidos nos inertes e a perda de material durante o processo de secagem. A retenção de sólidos ( $\mathrm{R})$ é definida como a razão entre a massa total de sólidos retida na superfície das partículas pela massa total de sólidos adicionada ao leito, Equação 2.

$$
R=\left(\frac{M_{a d}-M_{0}}{Q_{\text {pasta }} C_{s} t}\right) 100
$$

Onde: $M_{a d}=$ massa das partículas ao final do processo; $M_{0}=$ massa inicial das partículas.

A perda de material (P) foi determinada indiretamente a partir do balanço de massa, Equação 3.

$$
P=100-\varepsilon-R
$$

\section{RESULTADOS E DISCUSSÃO}

\subsection{Caracterização físico química das pastas}

As características físico-químicas das pastas de secagem são apresentadas na Tabela 2.

Tabela 2 - Características físico-químicas das pastas de secagem

\begin{tabular}{|c|c|c|c|c|c|}
\hline Pasta & pH & Reologia & Umidade (\% b.u.) & $\begin{array}{c}\text { Sólidos secos } \\
(\%)\end{array}$ & AA (mg/100g) \\
\hline A & 5,7 & Pseudoplástico & 57,1 & 42,9 & $1,1 \pm 0,11$ \\
\hline B & 5,5 & Pseudoplástico & 62,5 & 37,5 & $3,9 \pm 0,5$ \\
\hline C & 5,1 & Pseudoplástico & 69,6 & 30,4 & $7,5 \pm 2,4$ \\
\hline D & 4,7 & Pseudoplástico & 77,7 & 22,3 & $9,2 \pm 0,8$ \\
\hline E & 3,7 & Pseudoplástico & 81,2 & 18,8 & $10,3 \pm 1,3$ \\
\hline
\end{tabular}

A pasta A, cuja formulação contém $8 \%$ de polpa de uva, apresentou o maior valor de $\mathrm{pH}$, enquanto que a pasta $\mathrm{E}$, com $92 \%$ de polpa de uva, apresentou o menor $\mathrm{pH}$. O caráter ácido das pastas de secagem foi proporcional à quantidade de polpa de uva na formulação. Análises da polpa de uva e $\mathrm{SCH}$ realizadas separadamente apresentaram $\mathrm{pH}=3$ e $\mathrm{pH}=5,8$; respectivamente. Maior acidez, indicada pelo baixo $\mathrm{pH}$, pode ser favorável à estabilidade das antocianinas na pasta de secagem.

Todas as pastas estudadas apresentaram classificação reológica de fluido não newtoniano e comportamento pseudoplástico. O comportamento pseudoplástico das pastas pode ser atribuído à 
presença de $\mathrm{CH}$ na formulação.

\subsection{Ensaios de secagem} secagem.

$\mathrm{Na}$ Tabela 3 são apresentados os resultados do planejamento experimental dos ensaios de

Tabela 3 - Planejamento experimental e respostas do processo

\begin{tabular}{|c|c|c|c|c|c|c|c|}
\hline Ensaio & \multicolumn{3}{|c|}{ Variáveis } & \multicolumn{4}{c|}{ Respostas } \\
\hline $\mathrm{n}^{\circ}$ & $\mathrm{T}\left({ }^{\circ} \mathrm{C}\right)$ & $\mathrm{W}(\mathrm{mL} / \mathrm{min})$ & $\mathrm{SCH}(\%)$ & $\varepsilon(\%)$ & $\mathrm{R}(\%)$ & $\mathrm{P}(\%)$ & $\mathrm{AA}(\mathrm{mg} / 100 \mathrm{~g})$ \\
\hline 1 & 75 & 2,5 & 25 & 21,6 & 75,5 & 2,9 & $38,1 \pm 2,4$ \\
\hline 2 & 85 & 2,5 & 25 & 22,8 & 67,1 & 10,1 & $18,8 \pm 1,88$ \\
\hline 3 & 75 & 5,5 & 25 & 15,1 & 56,9 & 28,1 & $34 \pm 2,6$ \\
\hline 4 & 85 & 5,5 & 25 & 22,3 & 68,5 & 9,2 & $19,8 \pm 4,6$ \\
\hline 5 & 75 & 2,5 & 75 & 82,1 & 8,9 & 9,0 & $9,5 \pm 3,5$ \\
\hline 6 & 85 & 2,5 & 75 & 86,8 & 6,7 & 6,4 & $8,1 \pm 2,5$ \\
\hline 7 & 75 & 5,5 & 75 & 60,9 & 33,2 & 5,8 & $6,7 \pm 1,2$ \\
\hline 8 & 85 & 5,5 & 75 & 58,5 & 16,0 & 25,6 & $7,5 \pm 1,2$ \\
\hline 9 & 72 & 4 & 50 & 31,0 & 58,4 & 10,6 & $17,8 \pm 1,3$ \\
\hline 10 & 88 & 4 & 50 & 40,3 & 52,2 & 7,6 & $17,0 \pm 5,4$ \\
\hline 11 & 80 & 0,6 & 50 & 60,5 & 29,9 & 9,6 & $11,7 \pm 3,8$ \\
\hline 12 & 80 & 7,4 & 50 & 23,6 & 70,2 & 6,2 & $10,4 \pm 3,0$ \\
\hline 13 & 80 & 4 & 8 & 0,0 & 100,0 & 0,0 & - \\
\hline 14 & 80 & 4 & 92 & 83,3 & 9,6 & 7,1 & 0,00 \\
\hline 15 & 80 & 4 & 50 & 28,5 & 65,8 & 5,8 & $17,8 \pm 3,2$ \\
\hline 16 & 80 & 4 & 50 & 38,1 & 60,0 & 1,9 & $21,7 \pm 3$ \\
\hline 17 & 80 & 4 & 50 & 33,0 & 63,7 & 3,4 & $21,4 \pm 4,1$ \\
\hline
\end{tabular}

A partir dos resultados apresentados na Tabela 3 constata-se que a eficiência de produção de pó aumentou nos ensaios com elevadas frações de $\mathrm{SCH}$ na composição. Esse é um resultado satisfatório, já que o objetivo é produzir $\mathrm{CH}$ em pó com incorporação da uva. Analisando os ensaios 5 a 8 , composições com $75 \%$ de $\mathrm{SCH}$, nota-se que as maiores eficiências são observadas nos ensaios 5 e 6 , cuja vazão de atomização é de $2,5 \mathrm{~mL} / \mathrm{min}$.

A maior eficiência $(86,8 \%)$ foi alcançada em baixa vazão de atomização e elevadas condições de temperatura e composição de SCH na pasta. Em contrapartida, ensaios com baixas concentrações de SCH apresentaram baixas eficiências de secagem e elevada quantidade de material aderido (R) nas partículas, indicando que houve recobrimento das partículas inertes e não secagem da pasta. O ensaio 13, ponto axial do planejamento experimental e cuja composição da pasta foi de $92 \%$ de polpa de uva e $8 \%$ de SCH, não apresentou formação de pó, uma vez que ocorreu o colapso do regime dinâmico de jorro durante o processo de secagem.

Os resultados apresentados estão em concordância com os trabalhos de Medeiros et al. (2001) e 


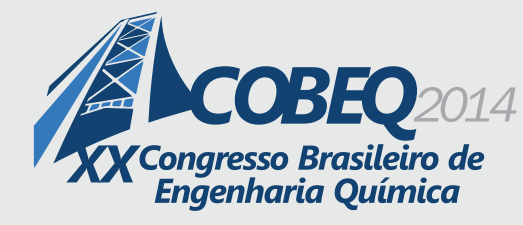

19 a 22 de outubro de 2014
Florianópolis/SC

Rocha et al. (2011). Os autores estudaram o efeito da alimentação de polpas de frutas com diferentes composições nas variáveis fluidodinâmicas de um leito de jorro, concluindo que algumas polpas interferiram favoravelmente na estabilidade dinâmica do leito, provocando intensa circulação de sólidos e vazões de jorro mínimo em níveis mais baixos, como é o caso da polpa com elevado teor de gordura, pectina e amido. Outras polpas, no entanto, interferiram negativamente, provocando problemas de instabilidade e diminuindo a eficiência, como as polpas com elevado teor de açúcares redutores.

A partir dos resultados observados neste trabalho, pode-se atribuir a boa eficiência de secagem à elevada composição de $\mathrm{CH}$ na pasta, que favoreceu a dinâmica de jorro no leito e consequentemente a eficiência na produção do pó. De forma análoga, a elevada proporção de polpa de uva (rica em carboidratos) na pasta de secagem está correlacionada com a forte aderência da pasta nas partículas inertes e consequente recobrimento das mesmas, conduzindo à baixa eficiência de processo.

Vale ressaltar que seis ensaios $(5,6,7,8,11$ e 14) apresentaram eficiência maior que 50\%, considerada satisfatória para o processo de secagem em leito de jorro. Contudo, os ensaios 11 e 14 correspondem a pontos axiais do planejamento experimental, sendo que o primeiro (11) apresenta a mais baixa vazão de atomização, enquanto que o segundo (13) apresentou produção de pó com composição isenta de antocianinas devido à elevada proporção de $\mathrm{SCH}$ na pasta. Considera-se, portanto, que os ensaios 5 a 8 apresentam boas condições operacionais de processo quanto à eficiência de secagem, sendo que os ensaios 5 e 6 foram considerados os pontos ótimos.

A Tabela 4 mostra os efeitos da (1) temperatura do ar de secagem, (2) vazão de atomização, (3) composição da pasta, bem como os efeitos de interação sobre a eficiência do processo.

Tabela 4 - Efeito das variáveis estudadas sobre a eficiência do processo

\begin{tabular}{|c|c|c|c|}
\hline Variável & Efeito & Desvio padrão & $p$ \\
\hline (1) Temperatura & 3,85 & 2,62 & 0,279100 \\
\hline (2) Vazão & $-17,39$ & 2,62 & 0,021884 \\
\hline (3) Composição & 50,77 & 2,62 & 0,002643 \\
\hline Interação 1 e 2 & $-0,29$ & 3,42 & 0,939590 \\
\hline Interação 1 e 3 & $-1,55$ & 3,42 & 0,693277 \\
\hline Interação 2 e 3 & $-10,61$ & 3,42 & 0,090006 \\
\hline
\end{tabular}

De acordo com a Tabela 4, o maior efeito $(50,77)$ está associado com a composição da pasta de secagem enquanto o efeito secundário $(-17,39)$ é dado pela vazão de atomização. Percebe-se que dentro do domínio experimental estudado só houve diferença significativa para os efeitos da vazão (2) e composição (3) para um intervalo de confiança de $95 \%(\mathrm{P}<0,05)$. O efeito de temperatura, bem como de interações não apresentaram diferença significativa.

No tocante ao teor de antocianinas do pó, constata-se a partir da Tabela 3 que as respostas também são dependentes da composição da pasta de secagem, como esperado. Maiores teores de antocianina foram de 38,1 mg/100g no ensaio 1 e $34 \mathrm{mg} / 100 \mathrm{~g}$ no ensaio 3, ambos com proporção de polpa de uva de $75 \%$ na pasta de secagem. Destaca-se ainda que apenas um ensaio, $\mathrm{n}^{\circ} 14$, não 


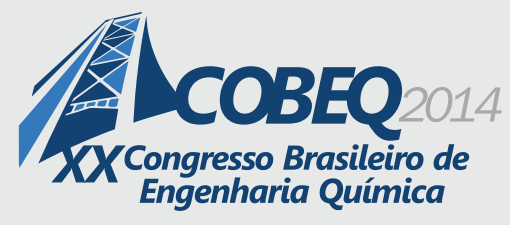

19 a 22 de outubro de 2014
Florianópolis/SC

apresentou incorporação de antocianinas, uma vez que a composição de polpa de uva de $8 \%$ se mostrou insuficiente para incorporação dos compostos no produto final. Nota-se que os resultados refletem o maior teor de antocianinas nas pastas com maior proporção de polpa de uva, como apresentado na Tabela 1.

Considerando os resultados satisfatórios em termos de eficiência de secagem, ensaios 5 e 6 , e o conteúdo de umidade médio do pó nestes ensaios, de 7,15\% (b.u), o conteúdo médio estimado de antocianinas por $100 \mathrm{~g}$ de sólido seco de pó é de 9,5 mg. Como a composição B, antes da secagem apresentou 10,4 mg de antocianinas em $100 \mathrm{~g}$ de sólido seco, avaliou-se uma degradação de apenas $8,6 \%$ durante o processo de secagem.

Os resultados de eficiência de produção de pó, e teor de antocianinas bem como de degradação são semelhantes aos encontrados por Braga e Rocha (2013). Os autores desenvolveram uma formulação em pó a base de leite e amora preta através da secagem em leito de jorro, alcançando a eficiência de obtenção de pó de até $60 \%$ e degradação de antocianinas menor que $15 \%$.

Os resultados apresentados neste trabalho demonstram que a secagem da mistura composta por $\mathrm{CH}$ com polpa de uva pode resultar em um alimento em pó inovador (na forma de alimento pronto para o consumo ou como ingrediente) de elevado valor agregado e nutricional, uma vez que preserva as antocianinas da própria fruta em um produto a base $\mathrm{CH}$, um suplemento alimentar já estabelecido e comercializado. As antocianinas tem a ingestão associada com a redução do risco de doenças cardiovasculares e cancerígenas (Neuhouser, 2004), enquanto que o colágeno hidrolisado é vendido com intuito na manutenção e reconstituição da pele, ossos, unhas e cabelos. Estudos também tem atribuído ao $\mathrm{CH}$ potencial terapêutico no tratamento de doenças como oesteoratrite e osteoporose (Moskowits, 2000; Bello e Oesser, 2006).

Destaca-se também a viabilidade técnica do processo, cuja eficiência superou $86 \%$ nas condições ótimas deste trabalho, visto que muitos problemas têm sido relatados durante a secagem de composições a base de polpas de frutas e/ou outros materiais higroscópicos e/ou termoplásticos, o que pode conduzir o processo a condições operacionais indesejadas, como a deposição de material sobre a câmara de secagem, e colapso do regime dinâmico, reduzindo a eficiência de secagem.

\section{CONCLUSÕES}

Este trabalho demonstra excelentes resultados de eficiência de produção de pó, de até $86,8 \%$ nas condições ótimas, durante a secagem de pasta composta por colágeno hidrolisado e polpa de uva em secador de leito de jorro. Este processo possibilitou alcançar quantidades significativas de antocianinas no produto final, de até $38,1 \mathrm{mg}$ de antocianinas por $100 \mathrm{~g}$ de pó, ou 9,5 mg/100g nas condições ótimas de eficiência de produção de pó. Dentro das condições experimentais observou-se que a composição da pasta de secagem foi a variável operacional com maior influencia sobre a eficiência de produção de pó, seguida pela vazão de atomização. Os resultados apresentados demonstram o desenvolvimento de um novo produto alimentício em pó composto por colágeno hidrolisado e polpa de uva com antocianinas agregadas e com elevado potencial de consumo na forma de um produto pronto ou como ingrediente para um alimento ou bebida. Adicionalmente verificou-se 
que o leito de jorro é uma boa alternativa para secagem de pastas formadas por colágeno hidrolisado e polpa de frutas, abrindo perspectivas para novos estudos de secagem de $\mathrm{CH}$ ou composições semelhantes incluindo produtos proteicos em leito de jorro.

\section{REFERÊNCIAS}

AOAC. Official Methods of Analysis of AOAC. USA: AOAC Inc., 2006.

BELLO, A. E.; OESSER, S. Collagen hydrolysate for the treatment of osteoarthritis and other joint disorders: a review of the literature. Curr. Med. Res. Opin. v. 22, n.11, p. 2221- 2232, 2006.

BRAGA, M.; ROCHA, S. C. S. Drying of milk-blackberry pulp mixture in spouted bed. T. Can. J. Chem. Eng., v. 91, n. 11, p. 1786-1792, 2013.

FANZONE, M.; ZAMORA, F.; JOFRÉ, V.; ASSOF, M.; PEÑA-NEIRA, A. Phenolic composition of malbec grape skins and seeds from Valle de Uco (Mendoza, Argentina) during ripening. Effect of cluster thinning. J. Agric. Food Chem., v. 59, n. 11, 6120-6136, 2011.

FULEKI, T.; FRANCIS, F. J. Quantitative Methods for Anthocyanins. J. Food Sci., v. 33, n. 1, p. $72-$ $77,1968$.

INSTITUTO ADOLFO LUTZ. Métodos físico-químicos para análise de alimentos. $4^{\mathrm{a}}$ ed. $1^{\mathrm{a}}$ edição digital. São Paulo: Instituto Adolfo Lutz, 2008.

MEDEIROS, M. F. D.; ROCHA, S. C. S.; ALSINA, O. L. S.; MEDEIROS, U. K. L.; DA MATA, A. M. L. Drying of pulps of tropical fruits in spouted bed: effect of composition on dryer performance. Dry. Technol., v. 20, n. 4-5, p. 855-881, 2002.

MOSKOWITZ, R. W. Role of collagen hydrolysate in bone and joint disease. Sem. Arthritis. Rheum., v. 30, n. 2, p. 87-99, 2000.

NEUHOUSER, M.L. Review: Dietary flavonoids and cancer risk: evidence from human population studies. Nutr. Cancer, v. 50, n. 1, p. 1-7, 2004.

WALRAND, S.; CHIOTELLI, E.; NOIRT, F.; MWEWA, S.; LASSEL, T. Consumption of a functional fermented milk containing collagen hydrolysate improves the concentration of collagenspecific amino acids in plasma. J. Agric. Food Chem., v. 56, n. 17, p. 7790-7795, 2008.

ROCHA, S. C. S.; SOUZA, J. S.; ALSINA, O. L. S.; MEDEIROS, M. F. D. Drying of Tropical Fruit Pulps: Spouted Bed Process Optimization as a Function of Pulp Composition. Dry. Technol., v. 29, n. 13, p. 1587-1599, 2011.

\section{AGRADESCIMENTOS}

Os autores agradecem a FAPESP pelo aporte financeiro necessário para a pesquisa. 